\title{
Políticas Públicas para mejorar la calidad de servicios de salud
}

\section{Resumen}

Juan Carlos Salas Padilla juancarlossalasp@hotmail.com

Universidad César Vallejo.

La presente investigación responde a la necesidad de que se considere Políticas Públicas de salud para mejorar la calidad del servicio de salud en los hospitales de servicio público. Esta gran necesidad se vio reflejada en la pandemia del covid 19 que nos ha demostrado que no puede haber desarrollo si se descuida la salud. Los objetivos que perseguimos en nuestra investigación es precisar la relación existente de los miles de trabajos de investigación en calidad de salud que deben de ayudar a los gobiernos en especial en el Perú, establecerse Políticas Públicas de salud para la mejora de la calidad del servicio de salud, demostrando que existe relación entre las políticas públicas y la mejora de la estructura la Gestión por Procesos de salud y la Gestión por Resultados en los Hospitales Públicos. Los resultados obtenidos podemos precisar que salvar vidas con atención de calidad debe ser la principal política pública del estado peruano.

Palabras claves: Calidad del servicio de salud, propuesta de mejora, Políticas Públicas. 


\title{
Public Policies to improve the quality of health services
}

\begin{abstract}
This research responds to the need to consider Public Health Policies to improve the quality of health service in public service hospitals. This great need was reflected in the covid 19 pandemic which has shown us that there can be no development if health is neglected. The objectives that we pursue in our research are to specify the existing relationship of the thousands of research works in quality of health that should help the governments, especially in Perú, to establish Public Policies of health for the improvement of the quality of the health service, demonstrating that there is a relationship between the public policies and the improvement of the structure of the Management by Processes of health and the Management by Results in the Public Hospitals. The results obtained show that saving lives with quality care must be the main public policy of the Peruvian state.
\end{abstract}

Keywords: Quality of health service, proposal for improvement, Public policies.

Artículo recibido: 25 dic. 2020 Aceptado para publicación: 26 ene. 2021 Correspondencia juancarlossalasp@hotmail.com Conflictos de Interés: Ninguna que declarar 


\section{"La mejora de calidad de servicio está en el talento humano (TH)"}

\section{Introducción}

En el transcurso de más de 54 años se ha venido estructurando la necesidad de que los gobiernos en América latina en forma especial logren establecer Políticas Públicas con enfoque al desarrollo de la calidad de salud, ya que en el trascurso del tiempo se han logrado desarrollar una gran diversidad de marcos teóricos de investigaciones a nivel mundial que apunta a lo mismo, la más acorde es la sostenida por el profesor y médico Donabedian, este gran maestro instituye en 1986 las dimensiones de la calidad del servicio de salud las mismas que las desarrolla como calidad en la estructura, proceso y resultado acompañado de indicadores quienes evaluaran a cada una de ellas, con respecto al trabajo de investigación señalado, se concentra en la ISO 9001: 2015 "sistemas de gestión de la calidad y la política nacional de modernización de la gestión pública y la gestión pública moderna; el primero regulado por International Organization for Standardization (ISO) 9001 (2015), norma concentrada en aquellos componentes y determinantes con la que necesariamente una estructura de la administración de calidad debe contar para lograr un sistema seguro que consienta dirigir y mejorar la calidad del servicio.

En la 23 a Conferencia Mundial de la Unión Internacional de Promoción de la Salud y de Educación para la Salud (UIPES) sobre Promoción de la Salud que se celebrará en Aotearoa (Nueva Zelanda): Promover la salud del planeta y el desarrollo sostenible para todos. La urgencia de que investigadores y profesionales del campo de la promoción de la salud adopten una visión ecológica de la salud y prioricen el desarrollo sostenible si aspiran a una promoción de la salud que siga siendo relevante, orientada a la prevención y que ponga freno a la mayor amenaza que se cierne sobre la salud de los seres humanos. en 1986 la Carta de Ottawa para la Promoción de la Salud, al declarar que los factores medioambientales pueden: favorecer la salud (de los seres humanos) o perjudicarla (Ratima, M., Martin, D., Castleden, H., \& Delormier, T. 2019, p. 102).

El sector Salud en el Perú, ha tomado protagonismo frente a esta pandemia Covid-19, donde se evidenció las defíciencias en todos los hospitales a nivel nacional, porque quienes han gobernado durante los últimos 30 años, poco o nada les ha interesado la gestión pública y gobernabilidad, para mejorar la calidad de los servicios de salud y esto se debe a la falta de líderes en el sector salud, que desconocen los procesos 
administrativos para poder llevar de forma exitosa los métodos que promuevan mejoras de los servicios salud, y satisfacer a los usuarios externos, que son muy complejos, pero que necesita darle un análisis profundo del tema; por otro lado cuando hablamos de calidad, este tiene un significado muy amplio para cada sujeto, para unos representa mejores cuidados y eficiencia y para otros trato digno (Gilmore y Moraes, 1996), este hecho hace que sea más complicado lograr ese equilibrio que contente a los usuarios.

Es importante señalar al respecto lo que nos dice Del Carmen Sara, J. C. (2019). los sistemas de salud de alta calidad pueden salvar más de 8 millones de vidas al año en los países de bajos y medianos ingresos, desafío que se traduce que en nuestro país se debe de revertir las graves inequidades en acceso y resultados de salud, que afecta a la población de menores recursos, generando nuevas estrategias que permitan brindar una mejor ruta de acceso a una atención integral y continua, en los diferentes niveles de atención, articulando e implementando mecanismos y arreglos institucionales que lo hagan posible (p. 291).

León (2016) afirma si existe una buena calidad de servicio los pacientes estarán satisfechos. En el mismo sentido Huarcaya (2015) determinar la importancia de la percepción de la calidad de atención recibida por los usuarios del servicio de emergencias, 1 sienten que existe una regular calidad es decir no satisfactorio en su totalidad, por lo que es necesario su mejora; Vásquez y López (2015) reafirmaron que hay en cada hospital una dimensión insatisfacción moderada percibida, y en otras deficiencias mayores.

Guamán y Sumba (2013) señalan que los usuarios perciben una atención excelente cuando un hospital cuenta con profesionales comprometidos e infraestructura segura; y desde la opinión del personal es un establecimiento que necesita de un mayor presupuesto para la adquisición de nuevos equipos y aumento de recurso humano con estabilidad laboral.

\section{ENFOQUE DE CALIDAD EN LOS SERVICIOS DE SALUD.}

El enfoque de las Politicas Públicas y la calidad se los servicios de salud estarán basadas en función de las múltiples definiciones de calidad del servicio de salud, Céspedes, Cortés y Madrigal (2011) nos dicen que consta en aplicar ciencia y la tecnología buscando así maximizar los beneficios a la salud evitando acrecentar a la par riesgos, por lo que hablar de calidad es sinónimo de lograr que los servicios que se brindan consigan un 
equilibrio adecuado de beneficios y riesgos, escrutando lograr una correcta atención la primera vez y ser mejorada la siguiente. la Organización Mundial de la Salud [OMS, 2015] precisa que una atención en salud capaz de identificar necesidades de los usuarios externos, que predestina los recursos necesarios a las mismas de forma pertinente y efectiva tal cual el conocimiento lo permite, logrando beneficiar al usuario, eso es hablar de calidad del servicio de salud. Donabedian (1966) "es el grado en que los medios más deseables se utilizan para alcanzar las mayores mejoras posibles en la salud" (p.12), es así que, analizando las diferentes concepciones encontradas, la más acertada es aquella dada por el mencionado. Según el cuadro adjunto:

\begin{tabular}{|c|c|c|c|c|}
\hline $\begin{array}{l}\text { Variable de } \\
\text { estudio }\end{array}$ & $\begin{array}{l}\text { Definición } \\
\text { Conceptual }\end{array}$ & $\begin{array}{c}\text { Definición } \\
\text { Operacional }\end{array}$ & Dimensión & Indicadores \\
\hline \multirow{13}{*}{$\begin{array}{l}\text { Calidad de } \\
\text { servicios de } \\
\text { salud }\end{array}$} & \multirow{13}{*}{$\begin{array}{l}\text { Es la percepción } \\
\text { proporcionada por el } \\
\text { usuario respecto a la } \\
\text { capacidad de un } \\
\text { producto o servicio } \\
\text { de adaptarse a lo } \\
\text { que el paciente } \\
\text { espera de él, siendo } \\
\text { el mayor bienestar } \\
\text { después y tras el } \\
\text { proceso de atención } \\
\text { médica. } \\
\text { Asimismo, es } \\
\text { aquella que } \\
\text { proporciona al } \\
\text { usuario el más } \\
\text { completo y máximo } \\
\text { bienestar } \\
\text { (Donabedian. 1980). }\end{array}$} & \multirow{13}{*}{$\begin{array}{l}\text { Se expresa en los } \\
\text { niveles de bueno, } \\
\text { regular y malo, de } \\
\text { acuerdo con el rango } \\
\text { de puntaciones } \\
\text { obtenidas mediante la } \\
\text { aplicación de la } \\
\text { Escala valorativa } \\
\text { Calidad de servicios } \\
\text { de atención al } \\
\text { usuario, a la } \\
\text { percepción de los } \\
\text { usuarios de los } \\
\text { servicios de atención } \\
\text { médica en un hospital }\end{array}$} & \multirow{4}{*}{ Estructura } & Ubicación \\
\hline & & & & Comodidad \\
\hline & & & & Seguridad \\
\hline & & & & Equipamiento \\
\hline & & & \multirow{4}{*}{$\begin{array}{l}\text { Gestión } \\
\text { por } \\
\text { Proceso }\end{array}$} & $\begin{array}{l}\text { Atención } \\
\text { oportuna }\end{array}$ \\
\hline & & & & $\begin{array}{l}\text { Competencia } \\
\text { profesional }\end{array}$ \\
\hline & & & & Comunicación \\
\hline & & & & Cortesía \\
\hline & & & \multirow{5}{*}{$\begin{array}{l}\text { Gestión } \\
\text { por } \\
\text { Resultados }\end{array}$} & Credibilidad \\
\hline & & & & Integralidad \\
\hline & & & & Continuidad \\
\hline & & & & Efectividad \\
\hline & & & & Satisfacción \\
\hline
\end{tabular}

Fuente; Elaboración propia.

Asimismo, la propuesta es fortalecida a través de la Política Nacional de Modernización de la Gestión Pública (2013) cuyo enfoque de un estado naciente al servicio de las personas y que satisfaga a los mismos (enfoque ciudadano) con un componente de la gestión pública orientada a los resultados, con la finalidad de trasfigurar insumos en productos (bienes y servicios públicos, normas, seguridad jurídica y regulaciones) que obtengan como resultado una mayor satisfacción de todos los ciudadanos certificando sus derechos y aun mínimo costo; ante lo expuesto se formuló el siguiente problema: ¿ ¿De 
qué manera las propuestas de Políticas Públicas contribuirán a mejoras la calidad de servicio de salud?

Marcelo Nazareno (2019). América Latina muestra elevadas desigualdades en salud. Su reducción solo es posible a través de políticas públicas redistributivas, cuya viabilidad depende de una opinión pública que reconozca estas desigualdades y que apoye las acciones redistributivas del Estado. A pesar de la relevancia, son escasos los trabajos en América Latina sobre percepciones de desigualdad y preferencias redistributivas en salud. A partir del análisis de una encuesta poblacional. Conforme lo demuestra la encuesta realizada en el Hospital I ESSALUD Yurimaguas 2021-2025, se realizó una muestra de estudio constituida por 300 usuarios externos de 18 a 80 años atendidos en el hospital de nuestra referencia para el análisis de la parte cuantitativa y para la parte cualitativa con entrevista focal dirigidas a usuarios externos e internos el Alfa de Cronbach, obteniéndose un coeficiente muy al alto ( $\square>0,988$ ) de atención regular que no garantiza una salud de calidad, presentándose las desigualdades de atención con las clínicas.

\section{RESULTADOS}

Las estrategias de búsqueda de datos para reforzar nuestra investigación SOBRE CALIDAD DE SERVICIO USANDO EL MODELO SERVQUAL, encontramos las siguientes más resaltantes:

\begin{tabular}{|c|c|c|}
\hline Autor & Objetivo del estudio & Resultados \\
\hline $\begin{array}{l}\text { Casalino - } \\
\text { Carpio (2008) }\end{array}$ & $\begin{array}{l}\text { Calidad de servicio de } \\
\text { la consulta externa de } \\
\text { Medicina Interna de } \\
\text { un hospital general de } \\
\text { Lima mediante la } \\
\text { encuesta Servqual } \\
\text { (Hospital Nacional } \\
\text { Arzobispo Loayza de } \\
\text { Lima, Perú }\end{array}$ & $\begin{array}{l}\text { Encontramos en total } 44,36 \% \text { de satisfacción y } \\
\text { satisfacción amplia. Asociado con satisfacción global se } \\
\text { encontró: sexo, grupo etario y nivel de instrucción; la } \\
\text { satisfacción en la dimensión tangible se encontró asociada } \\
\text { con el grupo etario; la satisfacción en la dimensión } \\
\text { confiabilidad estuvo asociada con el grupo etario, nivel de } \\
\text { instrucción, tiempo de llegada, zona de residencia y estrato } \\
\text { social; la satisfacción en la dimensión respuesta rápida } \\
\text { estuvo asociada con la zona de residencia; la satisfacción } \\
\text { en la dimensión seguridad, con el grupo etario y zona de } \\
\text { residencia; y, la satisfacción en la dimensión empatía } \\
\text { estuvo asociada con el grupo etario y nivel de instrucción. } \\
\text { Conclusiones: Las pacientes mujeres tuvieron mayores } \\
\text { niveles de insatisfacción global y en la dimensión } \\
\text { seguridad que los varones. La edad de } 18 \text { a } 20 \text { años fue un } \\
\text { factor asociado a mayores niveles de insatisfacción global } \\
\text { y con las dimensiones tangibles, confiabilidad, seguridad y } \\
\text { empatía. La edad mayor de } 65 \text { años fue un factor asociado } \\
\text { a satisfacción global y en las dimensiones tangibles, } \\
\text { confiabilidad, seguridad y empatía. La instrucción }\end{array}$ \\
\hline
\end{tabular}




\begin{tabular}{|c|c|c|}
\hline & & $\begin{array}{l}\text { secundaria o superior fue un factor asociado con } \\
\text { insatisfacción global y en la dimensión empatía. La } \\
\text { procedencia de zonas rurales se asoció con satisfacción } \\
\text { global amplia y en las dimensiones respuesta rápida y } \\
\text { seguridad }\end{array}$ \\
\hline$\frac{\text { Torres y Lastra }}{\underline{(2008)}}$ & $\begin{array}{l}\text { Propuesta de una } \\
\text { escala para medir la } \\
\text { calidad del servicio de } \\
\text { los centros de atención } \\
\text { secundaria de salud }\end{array}$ & $\begin{array}{l}\text { De acuerdo con los resultados de este estudio, se puede } \\
\text { concluir, que la escala propuesta para medir la Calidad del } \\
\text { Servicio de los centros de salud de atención secundaria, } \\
\text { desde la perspectiva de los pacientes, presenta un buen } \\
\text { grado de fiabilidad, validez y dimensionalidad. Esto es } \\
\text { especialmente relevante, considerando que actualmente en } \\
\text { los centros de atención secundaria de salud, no se han } \\
\text { desarrollado escalas de calidad del servicio que cumplan } \\
\text { estas condiciones. } \\
\text { En este análisis, el estadístico Alpha de Cronbach arrojó en } \\
\text { todos los casos valores muy superiores a 0,6, que es el } \\
\text { recomendado para un análisis exploratorio (Nunnally, } \\
\text { 1978; Hair et al., 1998) (confiabilidad 0,70, empatía 0,87, } \\
\text { capacidad de respuesta 0,73, accesibilidad 0,73 y tangibles } \\
0,82 \text { ). Sin embargo, la variable ACC5 perteneciente a la } \\
\text { dimensión accesibilidad fue eliminada del análisis, por } \\
\text { presentar una correlación de } 0,25 \text {, que está por debajo de } \\
0,3 \text { que es el mínimo establecido para este tipo de análisis } \\
\text { (Nurosis, 1993). }\end{array}$ \\
\hline$\frac{\text { Jélvez et al. }}{\underline{(2010)}}$ & $\begin{array}{l}\text { Evaluar la calidad de } \\
\text { servicio del Centro de } \\
\text { Salud Familiar en } \\
\text { Chile (centro de salud } \\
\text { Familiar Paulina A. } \\
\text { Pereda en Talcahuano, } \\
\text { Chile) }\end{array}$ & $\begin{array}{l}\text { La calificación promedio global para las expectativas en la } \\
\text { muestra de los pacientes encuestados resultó ser de } 4,28 \\
\text { puntos de un máximo de } 5 \text { puntos, es decir, ellos esperan } \\
\text { recibir un servicio muy bueno. La dimensión de } \\
\text { Confiabilidad muestra el nivel más alto de expectativa con } \\
4,31 \text { puntos en comparación con otras dimensiones. La } \\
\text { calificación promedio global para las percepciones en la } \\
\text { muestra de los pacientes encuestados resultó ser de } 3,86 \\
\text { puntos de un máximo de } 5 \text { puntos. La dimensión } \\
\text { Responsabilidad es la que, en promedio, tiene las } \\
\text { percepciones más bajas, alcanzando un valor de } 3,70 \\
\text { puntos, en tanto que la dimensión Empatía es la que } \\
\text { presenta la percepción promedio más alta alcanzando a } \\
3,98 \text { puntos. Finalmente, en base a la información lograda } \\
\text { se proponen las características que debería tener el servicio } \\
\text { que ofrece el Centro de Salud } \\
\text { Familiar. }\end{array}$ \\
\hline Lavín (2010) & $\begin{array}{l}\text { Administración del } \\
\text { capital de trabajo: un } \\
\text { enfoque estratégico de } \\
\text { calidad en la gestión } \\
\text { pública hospitalaria. } \\
\text { Evaluar la calidad de } \\
\text { los servicios de un }\end{array}$ & $\begin{array}{l}\text { En el factor trato al paciente y empatía, a pesar de que la } \\
\text { media de esta dimensión se caracterizó como un servicio } \\
\text { bueno en general, se identificó que un } 27.5 \% \text { señala que la } \\
\text { confianza que brinda el personal de hospital ABC a los } \\
\text { pacientes es incipiente, así mismo el } 26.5 \% \text { expresó que el } \\
\text { trato que se brinda al paciente va de pésimo a regular. La } \\
\text { dimensión de la capacidad de respuesta obtuvo una media }\end{array}$ \\
\hline
\end{tabular}




\begin{tabular}{|c|c|c|}
\hline & $\begin{array}{l}\text { hospital público de } \\
\text { segundo nivel en } \\
\text { Tamaulipas, México }\end{array}$ & $\begin{array}{l}\text { general de } 3.5 \text { determinándose en este estudio como el } \\
\text { factor con menor nivel de calificación por parte de los } \\
\text { usuarios. Al respecto se señala que un porcentaje } \\
\text { mayoritario (52.5\%) manifestó que el tiempo de espera en } \\
\text { la consulta externa es incipiente. El } 48.3 \% \text { de los usuarios } \\
\text { calificó de pésimo a regular el servicio de urgencias, } \\
\text { mientras que el } 43.3 \% \text { señaló que el tiempo de espera para } \\
\text { las consultas de especialidad requiere ser mejorado por el } \\
\text { hospital ABC. El } 24.2 \% \text { indicó que el número de } \\
\text { empleados para atender a los pacientes requiere atención } \\
\text { por parte de las autoridades sanitarias de esta unidad } \\
\text { hospitalaria, al calificar este indicador de pésimo a regular. } \\
\text { En el factor de efectividad y oportunidad, se encontró que } \\
\text { el } 39.2 \% \text { de los usuarios valoró de pésimo a regular el } \\
\text { surtimiento de los medicamentos y el tiempo que el } \\
\text { hospital ABC opera para los exámenes diagnósticos } \\
\text { (estudios de sangre, rayos x, entre otros); en esta dimensión } \\
\text { también se identificó que un } 23.3 \% \text { señaló que el estado de } \\
\text { las instalaciones va de pésimo a regular. En la dimensión } \\
\text { de tiempos de espera, se observó que independientemente } \\
\text { de que la media (3.7) de este factor se calificó como un } \\
\text { servicio bueno en general, cabe señalar que porcentajes } \\
\text { importantes de usuarios califican de incipiente la calidad } \\
\text { del servicio en esta dimensión. Por ejemplo, valoraron de } \\
\text { pésimo a regular los aspectos de tiempos de espera en } \\
\text { farmacia (el } 30 \% \text { ), el tiempo de espera para las } \\
\text { intervenciones quirúrgicas, así como el tiempo que tardan } \\
\text { para los resultados de los estudios socioeconómicos (el } \\
25 \% \text { de los encuestados así lo expresó). En el factor de } \\
\text { seguridad y limpieza, el } 25.8 \% \text { de los encuestados señala } \\
\text { que la limpieza es incipiente (calificada de pésima a } \\
\text { regular) y un } 22.5 \% \text { también calificó en este nivel de } \\
\text { calidad la seguridad dentro de las instalaciones del hospital } \\
\text { ABC. }\end{array}$ \\
\hline$\frac{\text { Regaira et al. }}{\underline{(2010)}}$ & $\begin{array}{l}\text { Evaluar la relación } \\
\text { entre calidad } \\
\text { asistencial y variables } \\
\text { sociodemográficas } \\
\text { (unidad de cuidados } \\
\text { intensivos de Navarra, } \\
\text { España) }\end{array}$ & $\begin{array}{l}\text { La puntuación de las percepciones }(66,92) \text { superó la de las } \\
\text { expectativas }(62,30) \text { y no guardó relación con las } \\
\text { características sociodemográficas. La puntuación media de } \\
\text { la diferencia entre percepciones y expectativas para el total } \\
\text { de la escala SERVQUAL fue de } 4,62 \text {; asimismo, fue } \\
\text { positiva para cada una de las dimensiones: } \\
\text { tangibilidad }=1,44 \text {, fiabilidad }=0,53 \text {, capacidad de } \\
\text { respuesta }=0,95 \text {, seguridad }=0,99 \text {, empatía }=0,71 \text {. No se } \\
\text { encontraron asociaciones estadísticamente significativas } \\
\text { entre la calidad asistencial evaluada por los pacientes y las } \\
\text { variables sociodemográficas. }\end{array}$ \\
\hline$\underline{\text { Calixto et al. }}$ & $\begin{array}{l}\text { ESCALA } \\
\text { SERVQUAL: } \\
\text { VALIDACIÓN EN }\end{array}$ & $\begin{array}{l}\text { La adaptación cultural y semántica fue realizada por cuatro } \\
\text { jueces peritos en el área, que evaluaron la equivalencia } \\
\text { conceptual y cultural de los elementos de la escala. La }\end{array}$ \\
\hline
\end{tabular}




\begin{tabular}{|c|c|c|}
\hline & $\begin{array}{l}\text { POBLACIÓN } \\
\text { MEXICANA (hospital } \\
\text { general de Celaya, } \\
\text { Guanajuato) }\end{array}$ & $\begin{array}{l}\text { consistencia interna del instrumento fue evaluada por el } \\
\text { test Alfa de Cronbach con resultado } \alpha=0,74 \text { en la escala } \\
\text { total y para cada una de las subescalas, siendo de } \mu=0,82 \\
\text { para expectativas y } \mu=0,66 \text { para percepciones, y análisis } \\
\text { factorial en que fueron detectadas las cinco dimensiones de } \\
\text { la escala, demostrando que la misma es válida y confiable } \\
\text { para medir el servicio de enfermería en la población } \\
\text { mexicana. }\end{array}$ \\
\hline $\begin{array}{l}\text { Cabello y } \\
\text { Chirinos (2012) }\end{array}$ & $\begin{array}{l}\text { Validación y } \\
\text { aplicabilidad de } \\
\text { encuestas } \\
\text { SERVQUAL } \\
\text { modificadas para } \\
\text { medir la satisfacción } \\
\text { de usuarios externos } \\
\text { en servicios de salud. } \\
\text { (hospital general de } \\
\text { Celaya, Guanajuato) }\end{array}$ & $\begin{array}{l}\text { El análisis factorial mostró que el } 88,9 \% \text { de la varianza de } \\
\text { las variables en CE y 92,5\% en E, explicaban los } 5 \\
\text { componentes o dimensiones de la calidad. El coeficiente } \\
\text { alfa de Cronbach fue } 0,984 \text { para CE y } 0,988 \text { para E. La } \\
\text { satisfacción global de los usuarios en CE fue } 46,8 \% \text { y } 52,9 \\
\% \text { en E. Las variables con mayor insatisfacción en CE } \\
\text { fueron: difícil acceso a citas, demora para la atención en } \\
\text { farmacia, caja, módulo SIS y el trato inadecuado; en } \\
\text { emergencia: deficiente mejora o resolución del problema } \\
\text { de salud, deficiente disponibilidad de medicinas y demora } \\
\text { para la atención en farmacia, caja y admisión. } \\
\text { Conclusiones: Las encuestas de CE y E, han demostrado } \\
\text { propiedades psicométricas para ser considerados como } \\
\text { instrumentos válidos, confiables y aplicables, para medir } \\
\text { el nivel de satisfacción de los usuarios externos, identificar } \\
\text { las principales causas de insatisfacción e implementar } \\
\text { proyectos de mejora. }\end{array}$ \\
\hline
\end{tabular}

Asi mismo otras dimensiones establecidas por EL MODELO SERVQUAL, encontramos

las siguientes más resaltantes:

\section{Definición conceptual de dimensiones de la calidad de servicios de salud}

\begin{tabular}{|l|l|l|}
\hline \multicolumn{1}{|c|}{$\begin{array}{c}\text { Nombre de } \\
\text { Dimensión }\end{array}$} & \multicolumn{1}{c|}{ Descripción } & \multicolumn{1}{c|}{ Fuente } \\
\hline Accesibilidad & $\begin{array}{l}\text { Evalúa las posibilidades de acceso que los usuarios } \\
\text { tienen sobre los servicios médicos en general }\end{array}$ & Torres y Lastra (2008). \\
\hline $\begin{array}{l}\text { Capacidad de } \\
\text { respuesta / } \\
\text { Respuesta } \\
\text { rápida }\end{array}$ & $\begin{array}{l}\text { Evalúa si la cantidad de empleados es suficiente para } \\
\text { la atención, así como los tiempos de espera para } \\
\text { poder recibir el servicio médico solicitado. }\end{array}$ & $\begin{array}{l}\text { Torres y Lastra 2008); } \\
\text { Regaira et al, (2010); } \\
\text { Casalino, (2008). }\end{array}$ \\
\hline Confiabilidad & $\begin{array}{l}\text { Seguridad Refiere a la competencia del personal, la } \\
\text { confianza en las revisiones y diagnósticos médicos } \\
\text { realizados, así como en los resultados de exámenes } \\
\text { que se brinda a los pacientes. Seguridad que el } \\
\text { paciente tiene de recibir el servicio que le fue } \\
\text { prometido. Es decir, prestar el servicio en el } \\
\text { momento preciso, cuando es requerido y de manera } \\
\text { correcta. }\end{array}$ & $\begin{array}{l}\text { Torres yastra (2008); } \\
\text { Casalino (2008); Regaira } \\
\text { et al.( 2010); Jélvez et al. } \\
\text { (2010); Castellano y } \\
\text { González, 2010. }\end{array}$ \\
\hline Empatía & $\begin{array}{l}\text { Medición del trato, atención e interés que el personal } \\
\text { de la institución hospitalaria manifiesta hacia los }\end{array}$ & $\begin{array}{l}\text { Torres y Lastra (2008); } \\
\text { Casalino (2008); Regaira et }\end{array}$ \\
\hline
\end{tabular}




\begin{tabular}{|l|l|l|}
\hline & $\begin{array}{l}\text { usuarios de la misma y el cuidado que se da de } \\
\text { manera individualizada. Para que se dé la empatía es } \\
\text { necesaria una buena comunicación entre la } \\
\text { organización y el usuario. }\end{array}$ & $\begin{array}{l}\text { al. (2010); Jélvez et } \\
\text { al.(2010). }\end{array}$ \\
\hline $\begin{array}{l}\text { Elementos } \\
\text { tangibles }\end{array}$ & $\begin{array}{l}\text { Eonsidera el estado físico y las condiciones del } \\
\text { servicios de salud, así como la apariencia del } \\
\text { personal que participa en la prestación del servicio. }\end{array}$ & $\begin{array}{l}\text { Montelongo y y yastra (2008); } \\
\text { Casalino (2008); Regaira et } \\
\text { al. (2010); Jélvez et al. } \\
(2010) ;\end{array}$ \\
\hline Fiabilidad & $\begin{array}{l}\text { Si el personal de la institución es comprensivo con } \\
\text { los pacientes y sus problemas de salud. }\end{array}$ & $\begin{array}{l}\text { Regaira et al. (2010). } \\
\text { Responsabilidad } \\
\text { ayudar a los pacientes }\end{array}$ \\
\hline
\end{tabular}

Fuente: Pedraza-Melo, Norma Angélica et Lavín-Verástegui, Jesús et González-Tapia, Anahí et

Bernal-González, Idolina (2014)

\section{DISCUSIÓN}

En estos últimos 54 años la calidad del servicio de salud se ha vuelto un tema de salud pública que preocupa al estado y en especial a quienes dirigen instituciones prestadoras de salud; es por esa razón que se realizó este estudio orientado al establecimiento de políticas públicas que se deben de establecer orientada a plantear una propuesta basada en los planteamientos estratégicos del ISO 90001: 2015 de Gestión de la Calidad y el enfoque de la Política Nacional de Modernización de la Gestión Pública para mejorar la calidad del servicio de salud del hospital, abordando como objetivo principal elaborar una propuesta de un plan de mejora, con el fin de aportar en el camino del avance positivo de los indicadores de salud. En cuanto a los resultado se aprecia que la mayoría de los usuarios externos califica como regular la calidad del servicio de salud; comparando estos resultados con Huarcaya (2015) y Vásquez y López (2015) los mismos que concluyen que los usuarios califican a la calidad del servicio de salud como insatisfactoria en su mayoría; Basándonos en la teoría de la calidad del servicio de salud se puntualiza como un nivel alto de excelsitud profesional, un exiguo riesgo para los usuarios, uso eficiente de los recursos y un alto grado de satisfacción (Donabedian, 1966), de acuerdo a los resultado, la escasa aprobación de la calidad del servicio de salud, es una alerta de que no se viene cumpliendo con los estándares de calidad. 


\section{CONCLUSIONES}

Es importante que el Estado Peruano deba de establecer una Política Pública basada en los resultados mostrados en la presente investigación, la calidad de los servicios de salud debe ser tomado como un tema prioritario a trabajar, ya que los tiempos actuales así lo está exigiendo, son las vidas humanas que deben llevar a mayores responsabilidades y estrategias que lleven a servicios oportunos y reales para hacer frente al covid $19 \mathrm{y}$ otras enfermedades que van a ir apareciendo por el tema de los fenómenos ambientales que nos está llegando.

\section{REFERENCIAS}

Boza, R. (2016). Percepción de la calidad que tienen los usuarios sobre la atención que brinda la Unidad de Cuidados Intensivos del Hospital Nacional de Niños Dr. Carlos Sáenz Herrera, durante febrero y marzo del año 2016 (tesis de maestría). Universidad Estatal a Distancia, San José, Costa Rica.

Cabello, Emilio, \& Chirinos, Jesús L. (2012). Validación y aplicabilidad de encuestas SERVQUAL modificadas para medir la satisfacción de usuarios externos en servicios de salud. Revista Medica Herediana, 23(2), 88-95. Recuperado en 25 de octubre de 2020, de http://www.scielo.org.pe/scielo.php?script=sci_arttext\&pid=S1018$\underline{130 X 2012000200003 \& \operatorname{lng}=\mathrm{es} \& \ln \mathrm{ln}=\mathrm{es}}$

Calixto-Olalde, Maria Gloria, Sawada, Namie Okino, Hayashida, Miyeko, Mendes, Isabel Amélia Costa, Trevizan, Maria Auxiliadora, \& Godoy, Simone de. (2011). Escala SERVQUAL: validación en población mexicana. Texto \& Contexto Enfermagem, 20(3), 526-533. https://doi.org/10.1590/S0104-07072011000300014

Carlos, C., Montelongo, Y., y Najera, M. (2010). La calidad del servicio de un centro de información. En: Conciencia Tecnológica. No. 40 (2010), p. 5-9.

Casalino, G. (2008). Calidad de servicio de la consulta externa de Medicina Interna de un hospital general de Lima mediante la encuesta Servqual. En: Revista de la Sociedad Peruana de Medicina Interna. Vol. 21, No. 4 (2008); p.143-152.

Castellano, S. y Gonzalez, P.(2010). Calidad de servicio en farmacias tradicionales y de autoservicio: Estudio de caso. En: Revista Venezolana de Gerencia. Vol. 15, No.52 (2010), p. 570- 590 
Céspedes, Y., Cortés, R. y Madrigal, M. (2011). Validación de un instrumento para medir la percepción de la calidad de los servicios farmacéuticos del Sistema Público de Salud de Costa Rica. Rev Costarric Salud Pública; 20(2): 75-82.

Chanduví , R. (2011). “La Nueva Gestión Pública. Evolución y tendencias en el Perú”. Revista Gestiopolis, Lima, Perú. Recuperado de http://www.gestiopolis.com/economia-2/la-nueva-gestion-publicaevoluciontendencias.htm\&gt.

Del Carmen Sara, J. C. (2019). [Guidelines and strategies to improve the quality of care in health services]. Revista Peruana de Medicina Experimental y Salud Publica, 36(2), 288-295. https://doi.org/10.17843/rpmesp.2019.362.4449

Donabedian A. (1966). Evaluation the Quality of Medical Care. The Milbank Memorial Fund Quarterly, 44 (3), 166 - 203.

Donabedian A. (1984). La Calidad de la atención médica: definición y métodos de evaluación. Salud Pública de México, 32(2), 248 -249.

Guamán, M. y Sumba, M. (2013). Propuesta de Estrategias para el mejoramiento de Calidad de los Servicios al usuario externo en la Unidad de Cuidados Intensivos del Hospital Vicente Corral (Tesis de Maestría). Universidad del Cuenca, Ecuador.

Hernández, R.; Fernández, C. y Baptista, P. (2016). Metodología de la investigación. (6ta ed.). Ciudad de México, México: Mc Graw Hill.

Huarcaya, R. (2015). Percepción de la Calidad de Atención en los Usuarios del Servicio de emergencias del Hospital I Santa Margarita de Andahuaylas (Tesis de Pregrado). Andahuaylas: Perú.

Jélvez, Riquelme y Gómez (2010). Evaluación de la calidad de servicio en centro de salud familiar en Chile. Horizontes Empresariales, 9 (2) (2010), pp. 51-72. http://revistas.ubiobio.cl/index.php/HHEE/article/view/2032

Lavín, (2010). Administración del capital de trabajo: un enfoque estratégico de calidad en la gestión pública hospitalaria. https://www.redalyc.org/pdf/2654/265433711006.pdf

León, L. (2016). Niveles de satisfacción y calidad de atención médica de los usuarios del servicio de consulta externa en el Hospital Regional Policía Nacional del Perú (Tesis de doctorado), Chiclayo: Perú. 
Luis Enrique Ibarra Morales \& Emma Vanessa Casas Medina (2015). Aplicación del modelo Servperf en los centros de atención Telcel, Hermosillo: Una medición de la calidad en el servicio. Contaduría y Administración by División de Investigación de la Facultad de Contaduría y Administración, Universidad Nacional Autónoma de México. http://www.cya.unam.mx/index.php/cya/article/view/10. https://doi.org/10.1016/S0186-1042(15)72153-4

Marcelo Nazareno (2019). Desigualdades en salud y opinión pública. Un análisis de las preferencias redistributivas en la provincia de Córdoba, Argentina. Revista Gerencia y Políticas de Salud. Redalyc: ORCID: http://orcid.org/0000-0002-5757-5986. http://www.redalyc.org/articulo.oa?id=54559086008..

DOI: https://doi.org/10.11144/Javeriana.rgps18-36.dsop.

Organización Internacional de Estandarización (2015). ISO 900:2015. Sistema de Gestión de la Calidad. (5ta ed). Ginebra. Suiza.

Organización Mundial de Salud, (2015). La calidad de la atención médica/auditoría. Recuperado de http://www.salud.gob.do/Documentos/Publicaciones/PUB_CalidadAtencionMedica Auditor_20120813.pdf.

Ratima, M., Martin, D., Castleden, H., \& Delormier, T. (2019). Voces indígenas y sistemas de conocimiento: promover la salud del planeta, la equidad en salud y el desarrollo sostenible ahora y para las generaciones futuras. Global Health Promotion, 26(3_suppl), 102-105. https://doi.org/10.1177/1757975919838730.

Regaira Martínez, E., Sola Iriarte, M., Goñi Viguria, R., Del Barrio Linares, M., Margall Coscojuela, M. A., \& Asiain Erro, M. C. (2010). La calidad asistencial en cuidados intensivos evaluada por los pacientes mediante la escala SERVQUAL. Enfermería Intensiva, 21(1), 3-10. https://doi.org/10.1016/j.enfi.2009.10.001

Secretaría de Gestión Pública (2012). Política Nacional de Modernización de la Gestión Pública al 2021. Presidencia del Consejo de Ministros. Lima, Perú.

Torres Moraga, Eduardo, \& Lastra Torres, Jorge. (2008). Propuesta de una escala para medir la calidad del servicio de los centros de atención secundaria de salud. Revista de Administração Pública, 42(4), 719-734. https://dx.doi.org/10.1590/S0034$\underline{76122008000400005}$ 
Vásquez, A. y López, A. (2015). Propuesta de Mejora de la Calidad de Servicio en el Policlínico Chiclayo Oeste Ubicado en la Ciudad de Chiclayo, durante el período 2013 (Tesis de maestría). Universidad Católica Santo Toribio de Mogrovejo, Chiclayo, Perú. 